\title{
Analytical Prediction of Damage Growth in Notched Composite Panels Loaded in Axial Compression
}

Carlos G. Dávila, Damodar R. Ambur, and David M. McGowan

NASA Langley Research Center

Hampton, VA

Paper AIAA-99-1435

40th AIAA/ASME/ASCE/AHS/ASC Structures, Structural Dynamics and Materials Conference

St. Louis, Missouri

April 12-15, 1999 
AIAA-99-1435

\title{
ANALYTICAL PREDICTION OF DAMAGE GROWTH IN NOTCHED COMPOSITE PANELS LOADED IN AXIAL COMPRESSION
}

\author{
Carlos G. Dávila* \\ Vehicle Technology Center - ARL \\ NASA Langley Research Center, Hampton, VA 23681 \\ and \\ Damodar R. Ambur ${ }^{\dagger}$ and David M. McGowan ${ }^{*}$ \\ NASA Langley Research Center, Hampton, VA 23681
}

\begin{abstract}
$\underline{\text { Abstract }}$
A progressive failure analysis method based on shell elements is developed for the computation of damage initiation and growth in stiffened thick-skin stitched graphite-epoxy panels loaded in axial compression. The analysis method involves a step-by-step simulation of material degradation based on ply-level failure mechanisms. High computational efficiency is derived from the use of superposed layers of shell elements to model each ply orientation in the laminate. Multiple integration points through the thickness are used to obtain the correct bending effects through the thickness without the need for ply-by-ply evaluations of the state of the material. The analysis results are compared with experimental results for three stiffened panels with notches oriented at 0,15 and 30 degrees to the panel width dimension. A parametric study is performed to investigate the damage growth retardation characteristics of the Kevlar stitch lines in the panels.
\end{abstract}

\section{Introduction}

Stitched graphite-epoxy structures have demonstrated a potential for reducing the weight and the cost of future commercial transport aircraft. Under the sponsorship of the NASA Advanced Composites Technology (ACT) Program, Boeing (formerly McDonnell Douglas) has developed an automated process to stitch entire wing cover panels together, including skins, stringers, and spar caps. This process eliminates the need for most fasteners and reduces both the cost and weight of the structure. One of the requirements for the development of a structural design is the demonstration of structural damage tolerance. This requirement is often demonstrated by testing a panel with a two-stringer-bay-wide notch. The response of notched wing panels made of stitched composite materials and loaded in compression or tension is not well understood. The present paper describes an experimentally validated analysis method that predicts the damage initiation and growth at the notch tip location. The method can be used to calculate the residual strength of stitched composite primary structures.

Progressive failure analyses are computationally intensive for two reasons. Firstly, the nonlinear continuation procedure used in the analysis requires small load steps to achieve solution convergence and to represent local load redistributions accurately. Secondly, small elements must be used in the regions of damage propagation to represent the proper stress gradients at the boundary of the damage zones. Therefore, progressive damage analyses have rarely been successfully demonstrated on large built-up structural components, especially those made of composite materials with thick cross-sections. The focus of this investigation is to develop an efficient progressive damage model for predicting the effect of damage on the response and residual strength of wingbox cover panels loaded in compression. For computational efficiency, the nonlinear analysis model is based on superposed shell elements. Superposition is used to separate the failure modes for each ply orientation and to avoid the expense of evaluating the

\footnotetext{
* Aerospace Engineer, Structural Mechanics Branch. Member, AIAA.

$\dagger$ Assistant Head, Structural Mechanics Branch. Associate Fellow, AIAA.

‡ Aerospace Engineer, Structural Mechanics Branch. Senior Member, AIAA.
}

Copyright (C) 1999 by the American institute of Aeronautics and Astronautics, Inc. No copyright is asserted in the United States under Title 17, U.S. Code. The U.S. Government has a royalty-free license to exercise all rights under the copyright claimed herein for Government Purposes. All other rights are reserved by the copyright owner. 
failure criteria at all material points of every ply in the laminate. Bending effects are included by using multiple independent integration points through the thickness of the laminate. The effect of Kevlar stitches on the propagation of the damage was also investigated by varying the fiber buckling allowable value at the stitch locations, and by comparing the numerical results with the experimental results. Three stiffened wing-box cover panel specimens were tested, and the experimental results are compared with the analytical predictions.

\section{Specimen Configuration and Experimental Procedure}

The three-stringer panels used for this study were cut from wing-box cover panels manufactured by Boeing as part of the NASA ACT program. A complete description of this wing-box and the tests that were conducted earlier at NASA Langley Research Center can be found in Ref. 1 . The panels are 43 inches long and 19 inches wide, as shown in Fig. 1. The loaded ends of the panel are potted in an epoxy material, and knife-edge supports are provided on the unloaded edges to prevent premature panel buckling. A 7-inch-long, 0.1875 -inch-wide notch was cut at the center of the panel through the skin and the middle stiffener. The angle $\alpha$ of the cut is 0,15 , and 30 degrees for each of the panels tested, and these panels are designated as P-0, $\mathrm{P}-15$, and P-30, respectively.

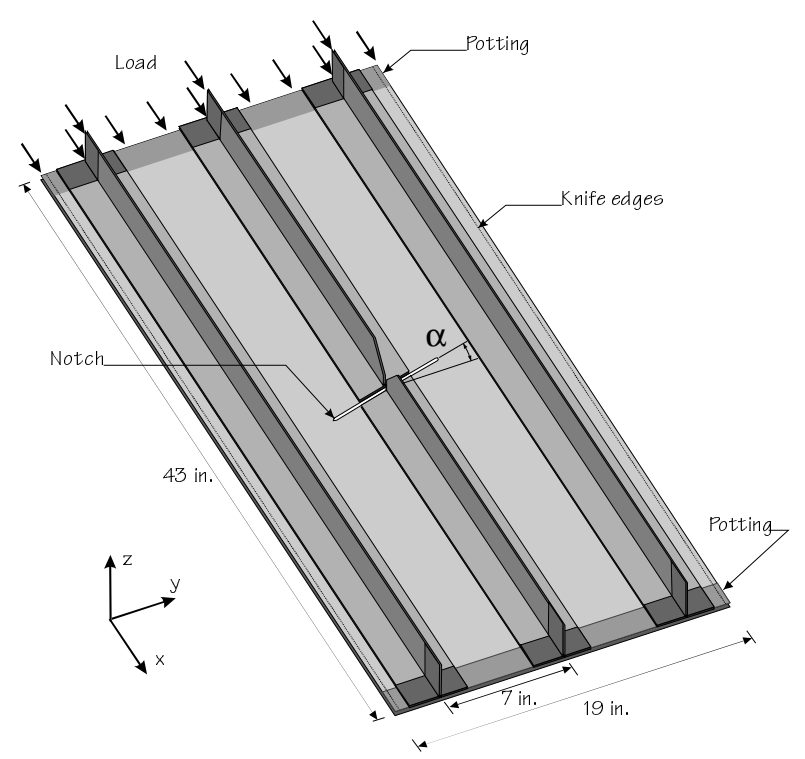

Figure 1. Geometry of notched wing-box cover panel subjected to axial compression load.

Since sufficient undamaged material for three identical panels was not available from the Boeing wing-box, panel P-0 was cut from the upper cover of the box, and panels P-15 and P-30 were cut from the lower cover of the box. The material system for all panels consists of stacks of AS4/3501-6 graphite-epoxy material, except for the 0-degree plies in the skin of panels P-15 and P-30, which consist of IM7/3501-6 graphite-epoxy material. A stack of material is approximately 0.058 inches thick, and is composed of 7 loosely tied plies with a stacking sequence of $[45 /-45 / 0 / 90]_{s}$. The thicknesses of the plies are $0.00633,0.01285$, and 0.007018 inches for $\pm 45,0$, and 90-degree orientations, respectively. The skin of panel $\mathrm{P}-0$ is composed of 8 stacks of material, for a total average thickness of 0.46 inches. The skins in panels $\mathrm{P}$ 15 and P-30 contain 9 stacks of material each, and, due to differences in compaction, their average ticknesses are 0.535 and 0.576 inches, respectively. The stiffeners in all panels are composed of 8 stacks of material and have an average thickness of 0.46 inches. The undamaged material moduli and strengths for the materials used in panels P-0, P-15, and P-30 can be found in Table 1. The strength values reported in this Table were obtained by dividing the design allowable values in Reference 2 by 0.85 , which is the margin of safety built into the allowable values by the manufacturer. The thickness fraction is the sum of the thicknesses of all of the plies with a given orientation divided by the total thickness of the laminate.

Table 1. Material constants for panels P-0, P-15, and $\mathrm{P}-30$ from the Boeing wing-box.

\begin{tabular}{|c|c|c|c|c|}
\hline \multirow[b]{2}{*}{ Ply orientation } & \multicolumn{3}{|c|}{ AS4/3501-6 } & \multirow{2}{*}{$\frac{\text { IM7/3501-6 }}{0}$} \\
\hline & 45 & $\mathbf{0}$ & 90 & \\
\hline \multicolumn{5}{|l|}{ Moduli } \\
\hline $\mathbf{E}_{\mathrm{L}}(\mathbf{M s i})$ & 15.04 & 15.30 & 14.87 & 15.04 \\
\hline $\mathbf{E}_{\mathrm{T}}(\mathbf{M s i})$ & 1.60 & 1.60 & 1.60 & 1.60 \\
\hline $\mathbf{G}_{\mathrm{LT}}(\mathbf{M s i})$ & 0.8 & 0.8 & 0.8 & 0.8 \\
\hline$v_{12}$ & 0.34 & 0.34 & 0.34 & 0.34 \\
\hline Thickness fraction & 0.2175 & 0.443 & 0.122 & 0.443 \\
\hline \multicolumn{5}{|l|}{ Strength } \\
\hline $\mathbf{X}_{t}(\mathbf{k s i})$ & 214. & 240. & 198. & 232. \\
\hline $\mathbf{X}_{\mathrm{c}}(\mathbf{k s i})$ & 164. & 164. & 164. & 160. \\
\hline $\mathbf{S}_{\mathrm{c}}(\mathbf{k s i})$ & 20.6 & 20.6 & 20.6 & 20.6 \\
\hline $\mathbf{Y}_{t}(\mathbf{k s i})$ & 5.88 & 5.88 & 5.88 & 5.88 \\
\hline $\mathbf{Y}_{\mathrm{c}}(\mathbf{k s i})$ & 36.5 & 36.5 & 36.5 & 36.5 \\
\hline
\end{tabular}

\section{$\underline{\text { Analysis Procedure }}$}

\section{Failure Analysis}

Failure modes in laminated composite panels are strongly dependent on panel geometry, loading 
direction, and ply orientation. There are four basic failure mechanisms that can induce compression failure in a laminated composite panel. These mechanisms are: matrix tensile or compressive cracking; fiber-matrix shearing; fiber buckling; and delamination. In order to simulate the damage growth accurately, the failure analysis must be able to predict the failure mode in each ply, and then apply the corresponding reduction in material stiffness as the loading level is increased. The compression failure criteria applied in the present analysis are those for unidirectional fiber composite materials as proposed by Hashin, ${ }^{3}$ with the elastic stiffness degradation models developed for compression by Chang et al. ${ }^{4}$ Unidirectional failure criteria are used and the stresses are computed in the principal directions for each ply orientation. The failure criteria included in the present analysis are summarized in the equations below. For each criterion, failure occurs when the failure index exceeds 1.0.

Matrix failure in tension and compression occurs due to a combination of transverse stress $\sigma_{y}$ and shear stress $\sigma_{x y}$. The failure index $e_{m}$ can be defined in terms of these stresses and the strength parameter $Y$ and the shear allowable value $S_{c}$. The matrix allowable value $Y$ takes the values of $Y_{t}$ in tension and $Y_{c}$ in compression. Failure occurs when the index exceeds 1.0. Assuming a linear elastic response, the failure index has the form:

$$
e_{m}=\sqrt{\left(\frac{\sigma_{y}}{Y}\right)^{2}+\left(\frac{\sigma_{x y}}{S_{c}}\right)^{2}}
$$

Fiber-matrix shearing failure occurs due to a combination of fiber compression and matrix shearing. The failure index has the form:

$$
e_{f}=\sqrt{\left(\frac{\sigma_{x}}{X_{c}}\right)^{2}+\left(\frac{\sigma_{x y}}{S_{c}}\right)^{2}}
$$

where $X_{c}$ is the fiber compression allowable value.

Fiber buckling occurs when the maximum compressive stress in the fiber direction exceeds the fiber buckling strength $X_{c}$, independent of the other stress components. The failure index for this mechanism has the form:

$$
e_{b}=-\frac{\sigma_{x}}{X_{c}}
$$

To simulate the failure modes, the elastic properties are made to be linearly dependent on three field variables, FVI through FV3. The first field variable represents the matrix failure, the second represents the fiber-matrix shearing failure, and the third represents fiber buckling. The values of the field variables are set equal to zero for the undamaged state. After a failure index has exceeded 1.0, the associated user-defined field variable is set equal to 1 . The associated field variable then continues to have the value of 1 , even though the stresses may be reduced significantly. This procedure ensures that the material does not "heal" after it has become damaged. The mechanical properties in the damaged area are reduced appropriately, according to the property degradation model defined in Table 2 . For example, when the matrix failure criterion is exceeded, the field variable $F V 1$ takes the value of 1 , and by the interpolation rule defined in Table 2, the transverse shear modulus $E_{y}$ and the Poisson's ratio $v_{x y}$ are set equal to zero. The field variables can be made to transition from 0 (undamaged) to 1 (fully damaged) instantaneously or as any specified function of the failure indices. Chang's degradation model is used in the present study, and the transition is assumed to be instantaneous.

The finite element implementation of this failure analysis was developed for the ABAQUS structural analysis program using the USDFLD user-written subroutine. $^{5-6}$ The program calls this routine at all material points of elements that have material properties defined in terms of the field variables. The subroutine provides access points to a number of variables such as stresses, strains, material orientation, current load step, and material name, all of which can be used to compute the field variables. Stresses and strains are calculated at each incremental load step, and evaluated by the failure criteria to determine the occurrence of failure and the mode of failure.

Table 2. Dependence of the material elastic properties on the field variables.

\begin{tabular}{lccccccc}
\hline \hline \multicolumn{1}{c}{ Material state } & \multicolumn{3}{c}{ Elastic Properties } & & $\boldsymbol{F V 1}$ & $\boldsymbol{F V 2}$ & $\boldsymbol{F V 3}$ \\
\hline No failure & $\mathrm{E}_{\mathrm{x}}$ & $\mathrm{E}_{\mathrm{y}}$ & $v_{\mathrm{xy}}$ & $\mathrm{G}_{\mathrm{xy}}$ & 0 & 0 & 0 \\
Matrix failure & $\mathrm{E}_{\mathrm{x}}$ & 0 & 0 & $\mathrm{G}_{\mathrm{xy}}$ & 1 & 0 & 0 \\
$\begin{array}{l}\text { Fiber/matrix } \\
\text { shear }\end{array}$ & $\mathrm{E}_{\mathrm{x}}$ & $\mathrm{E}_{\mathrm{y}}$ & 0 & 0 & 0 & 1 & 0 \\
Fiber buckling & 0 & 0 & 0 & 0 & 0 & 0 & 1 \\
\hline \hline
\end{tabular}

As was described previously, the material degradation model applied in this investigation uses the failure indices to determine the state of the material. For a particular failure mode and integration point, the state can either be intact or failed, without an intermediate state. This abruptness in change of state is noticeable in the strain history of any typical element in the path of the damage zone, as illustrated in Fig. 2. For a strain of approximately $-6,000 \mu \mathrm{in} / \mathrm{in}$, the high-strain field associated with the approaching damage zone can be observed to reach the element, and the strain 
becomes highly nonlinear. However, this nonlinearity does not indicate failure, since the nominal strain allowable value obtained from coupon tests is approximately $-15,000 \mu \mathrm{in} / \mathrm{in}$. Furthermore, numerical tests indicate that the strain allowable value necessary for optimal correlation with experimental results is higher than the nominal material allowable value by some factor $F A$. The strength scale factor $F A$ appears to be dependent upon laminate thickness. The values of $F A$ used for panels P-0, P-15, and P-30 are shown in Table 3.

Several factors contribute to the increase in apparent strength. First, the material that is adjacent to the damage front can stabilize the most highly loaded fibers and delay the onset of fiber buckling. Second, some small-scale delaminations may be present through the thickness, which could result in local load redistribution and, hence, reduced stresses. Finally, material nonlinearity in the model prior to and during failure, which is not represented in the present model, could be another contributor to the difference between the nominal allowable value and the one needed for optimal correlation with the experiment.

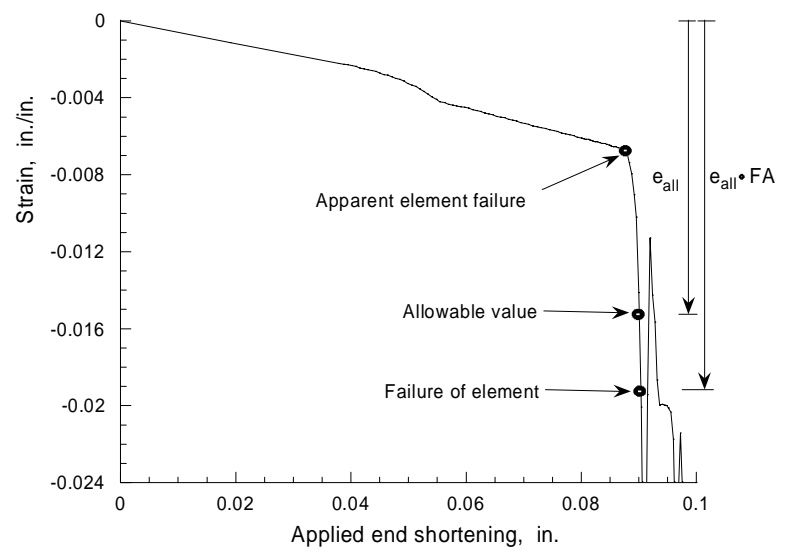

Figure 2. The strain at a typical material point along the damage path.

Table 3. Strength scale factor, $F A$.

\begin{tabular}{lccc}
\hline \hline & & Panel \\
& P-0 & P-15 & P-30 \\
\hline Skin thickness, in. & 0.430 & 0.535 & 0.576 \\
Strength scale factor, FA & 1.25 & 1.45 & 1.60 \\
\hline \hline
\end{tabular}

\section{Modeling Damage with Superposed Shell Elements}

A progressive damage model for notched laminates under tension was first proposed by Chang et al. ${ }^{7}$ The model accounts for all of the possible failure modes in each ply except delamination. Chang et al. ${ }^{4}$ later investigated the damage tolerance of composite materials subjected to compressive loads. The present analysis, which also deals with compression loads, is largely based on the latter work by Chang. However, the present analysis extends Chang's method from twodimensional membrane effects to a shell-based analysis that includes bending. Other researchers [e.g., Refs. 810] have performed plate and shell-based progressive failure analyses by applying a material degradation model at every material point in every ply in the laminate. In these investigations, the plate $[A, B, D]$ stiffness matrices are computed from the degraded ply properties. The disadvantage of this method is that the number of material points through the thickness that must be evaluated can be large, even for relatively thin laminates, and can significantly increase the computational effort. For the thick laminates used in the wing-box cover panels, the computational cost of this method may be prohibitive.

To improve the computational efficiency of the analysis, a method based on element superposition was developed that separates the failure modes for each ply orientation and does not rely on the computation of the $[A, B, D]$ matrices. The modeling is done such that the regions ahead of the notch tips, where a potential for damage growth is anticipated, are constructed of four superposed layers of shell elements that share the same nodes. No wall offset is applied to any of the elements. Each layer of elements represents one ply orientation (either $0,45,-45$ or 90 degrees), and each element spans the entire thickness of the laminate. It is implied that the plies for each orientation are uniformly distributed and can be smeared over the thickness of the laminate. Considering that the skin laminate is composed of either 8 or 9 stacks of material (56 or 63 plies), this assumption is appropriate. The elements used in the analyses consist of the ABAQUS four-node reducedintegration shear deformable S4R5 element (Ref. 5).

To obtain the correct stiffnesses corresponding to a given damage state, reduced engineering properties are applied to each layer. A reduced material property for a given orientation is simply the product of the engineering property and the sum of the thicknesses of all the plies in that orientation divided by the total laminate thickness. Reduced material properties are denoted by the notation []$_{R}$, as illustrated in Fig. 3 . Bending effects are taken into account by the use of five integration points through the thickness of the laminate. For all practical thick laminates, this reduction in number of integration points greatly reduces the computational complexity of an analysis. The 
separation of the material orientations also allows the use of stress and material axes defined along the fiber direction, which facilitates the computation of failure modes based on unidirectional material properties.

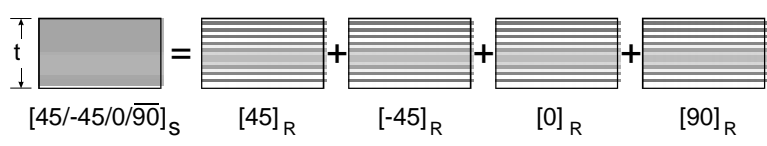

Figure 3. The thick laminate is modeled with four layers of superposed shell elements.

\section{Finite Element Analysis and Convergence Difficulties}

It was found during this investigation that the material degradation that is introduced into the model during the analysis can cause severe convergence difficulties. One problem was the sudden appearance of negative eigenvalues, past which convergence could not be obtained. The eigenmodes corresponding to these negative eigenvalues are always localized in the damage area, and correspond to either out-of-plane local buckling, as shown in Fig. 4, or inplane "hourglassing" deformations. Disabling the most distorted of the failed elements within 0.1 inches of the undamaged notch tip alleviated these difficulties. In addition, the panel was loaded under end shortening displacement control instead of force control in order to achieve convergence past the maximum load.

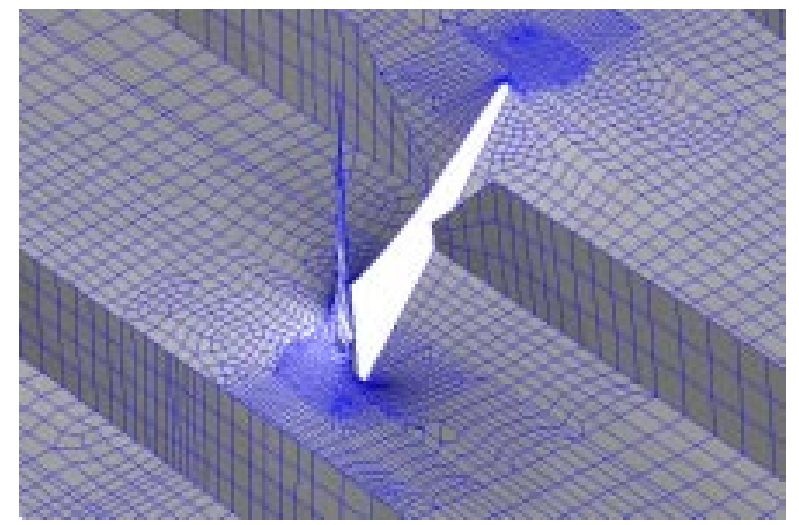

Figure 4. Typical eigenmode corresponding to a negative eigenvalue localized in the damage region.

A second convergence difficulty was related to the quality of the solution. It was found that, due to the stitching or other geometric discontinuities such as the edge of the stiffener flange, the damage zone does not always grow stably, but rather by discontinuous steps. Accurate solutions were obtained by forcing an average of approximately 100 load step increments per inch of damage growth through direct displacement control at the loaded edges of the panel. The region of damage growth is discretized such that the elements have a length of approximately 0.04 inches. The number of degrees of freedom ranges from 24,000 for the quartersymmetry model of panel P-0, to approximately 55,000 for panels P-15 and P-30. The total CPU time for an analysis ranges from 2 to 6 hours on a DEC Alpha workstation.

\section{Discussion of Test and Analysis Results}

\section{Panel $P-0$}

Panel P-0, with a 0-degree notch, was the first panel to be tested. The five strain gages shown in Fig. 5 were placed in the vicinity of the notch tip to monitor the growth of the damage zone. The first four gages, G1-G4, are in the path of the damage, while gage G5 is in the load path of gage G1. The experimental results shown in Fig. 6a indicate that damage initiation occurs at an applied end shortening displacement of $0.050 \mathrm{in.}$ This point is labeled " 0 " on the figure. At an applied displacement of 0.053 inches, the damage zone grows into the area of gage G1, as evidenced by the large increase in (negative) strain. This damage growth is confirmed by the data from gage G5, which indicates a reduction in the load as a consequence of the damage propagation. No further damage is measured until an end shortening displacement of 0.08 inches, when damage growth is indicated again through a large increase in strain at gage G2 (Point 2 on the figure). Simultaneously, load redistribution causes another reduction in strain at gage G5. For an end shortening displacement between 0.08 and 0.09 inches, there is again an indication of containment of the damage zone. At 0.09 inches, the strain at gage G3 increases, suggesting yet another growth in the damage zone.

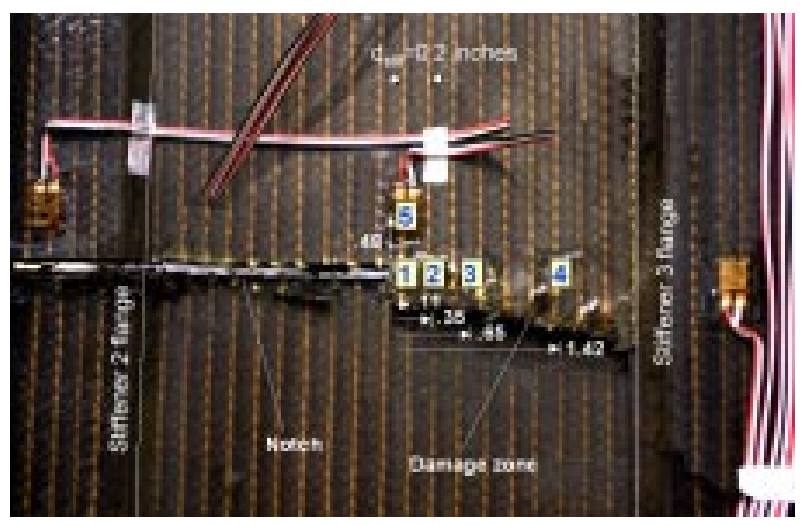

Figure 5. Location of strain gages G1-G5 in the region ahead of the notch tip. 


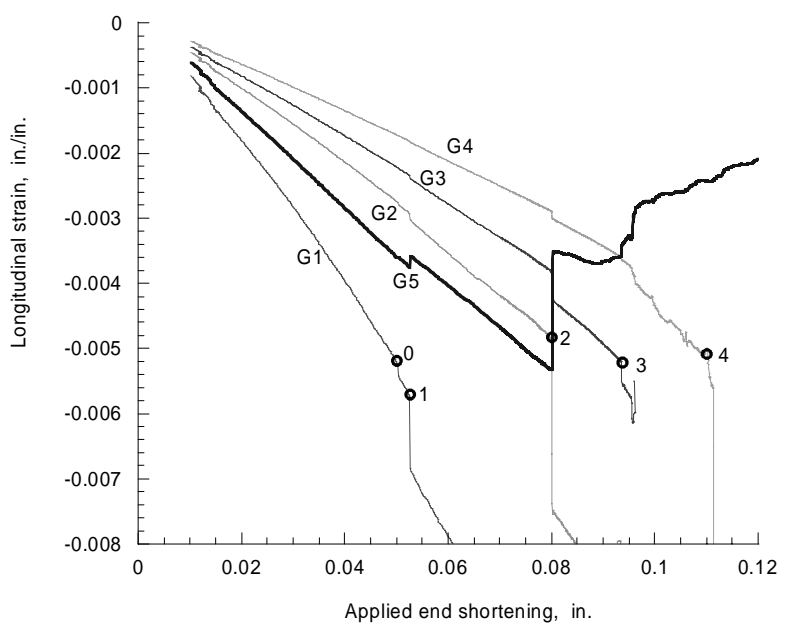

a. Measured strains for gages G1-G5.

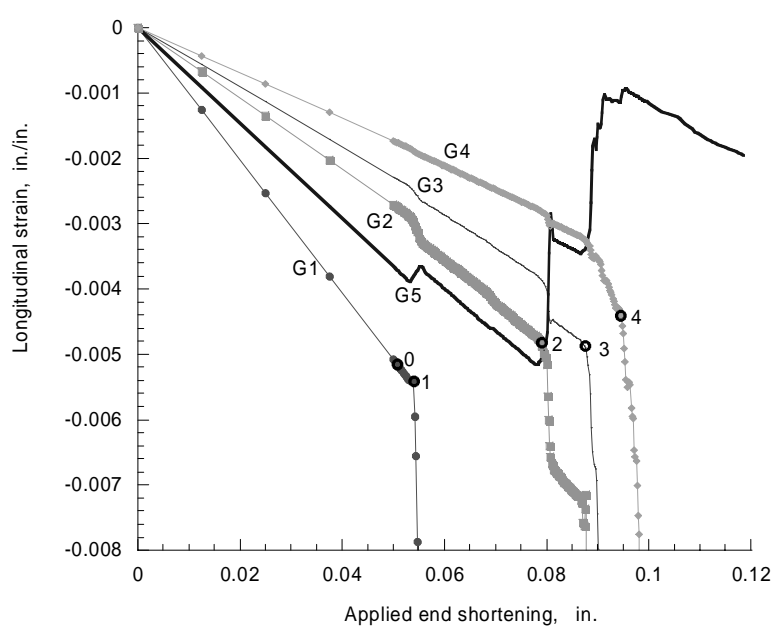

b. Computed strains for gages G1-G5.

Figure 6. Measured and computed strains at gage locations G1-G1 for panel P-0 as a function of applied end shortening displacement.

It is observed from the experimental results that the damage zone reaches each strain gage at a strain value of approximately $-5300 \mu \mathrm{in} . / \mathrm{in}$. It was concluded from the analysis that the discrete increments in damage growth occur due to the stitch lines increasing the fiber buckling strength locally by tying all of the plies in the laminate together. For this panel, the stitch lines run perpendicular to the notch direction and have a spacing of approximately 0.2 inches, as can be seen in the photograph in Fig. 5.

The finite element analysis results shown in Fig. 6b have the same characteristics as the test results: discrete damage growth at applied end shortening displacements of $0.053,0.08$ and 0.09 inches. The symbols in Fig. 6b correspond to converged solutions. The observed unloading response of gage G5 is properly represented by the analysis throughout the loading range considered.

The local reinforcing effect of the stitches was simulated in the finite element model by multiplying the fiber buckling allowable value by a stitch factor $S F$ that is determined empirically. Only the 0 -degree plies of the elements at the locations of the stitch lines were modified in this manner. It can be observed from the results shown in Fig. 7 that the strains at gage G5 are strongly affected by the stitch factor, with an increase in the value of the stitch factor generally resulting in the increased ability of the panel to support strain. It was determined that selecting $S F=2.3$ provides a good correlation between the analytical and experimental results. The distributions of the failure modes ahead of the notch tip are shown in Fig. 8 for the three ply orientations of 0,45 , and 90 degrees, and at an applied end shortening displacement of 0.112 inches. Darkcolored elements are designated as failed by the analysis for a particular mode and ply orientation. It can be observed that the fiber-shear mode is the dominant failure mode in the 0-degree plies, and that the matrix failure mode dominates in the 45 and 90 -degree plies.

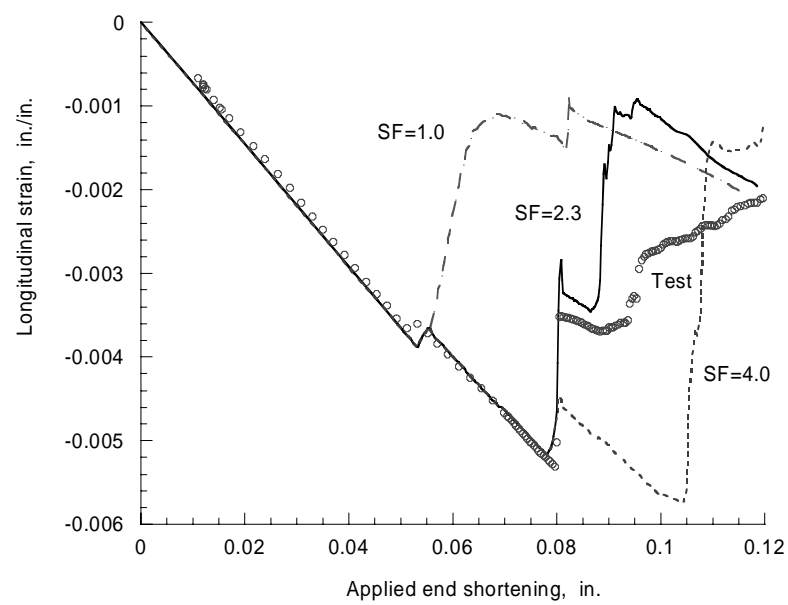

Figure 7. Effect of stitch factor $S F$ on strains at gage location G5. 


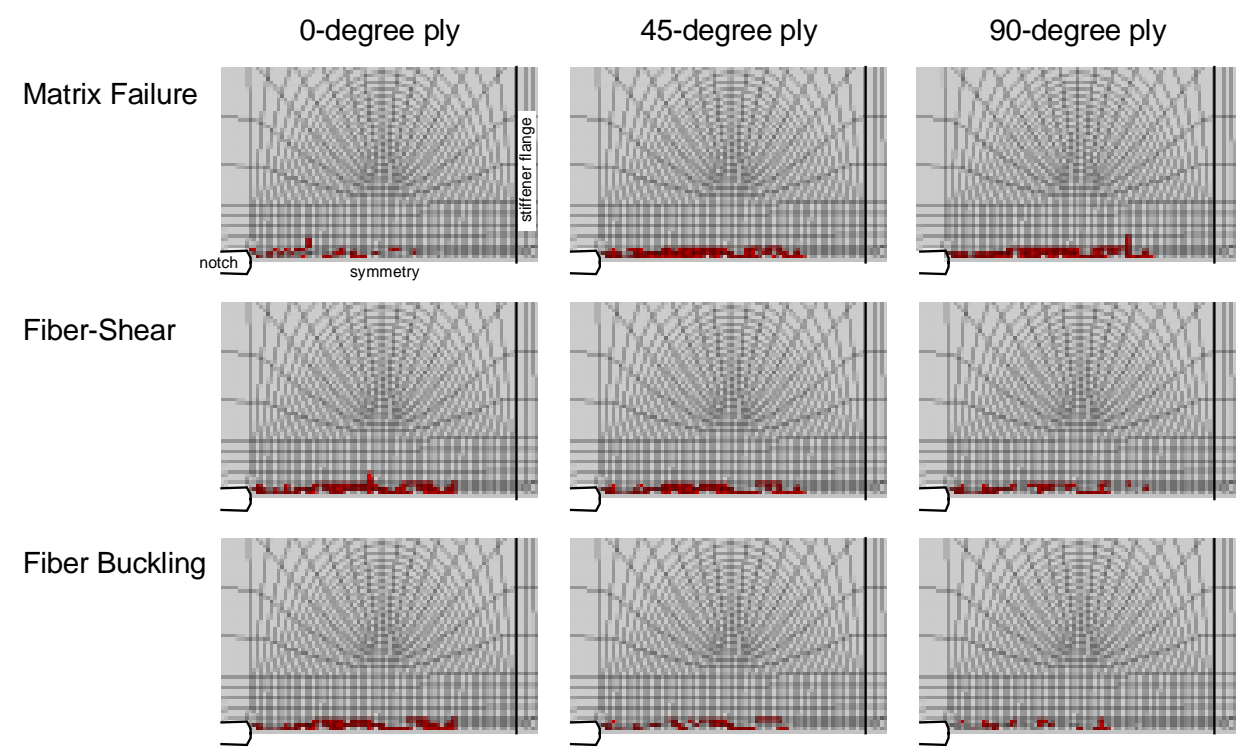

Figure 8. Distribution of failures by mode and ply orientation on the stiffener side of panel P-0 for an end shortening displacement of 0.112 inches.

\section{Panel P-15}

The dimensions of panel P-15 are identical to those of panel P-0. The differences between the panels are the following: 1) the notch is cut at 15 degrees instead of 0 degrees; 2) the 0-degree fibers in the skin are IM7 as opposed to AS4; 3) the skin is composed of 9 stacks of material rather than 8; and 4) the spacing between stitch lines is 0.5 inches instead of 0.2 inches. The experimental and predicted strains at the gage locations closest to the notch tips (see Fig. 9) are shown in Fig. 10. The longitudinal strain for gage G1R becomes nonlinear at approximately $-5500 \mu \mathrm{in} . / \mathrm{in}$., which is consistent with the results for panel P-0. Note that the placement of gage G1L is not exactly symmetric to gage G1R and that the strain recorded by gages G1L is somewhat smaller than that of G1R. It can also be observed that stitch lines are close to gages G2R and G3R and, as a consequence, the point at which their strain measurement results become nonlinear is somewhat lower than that of the other gages.

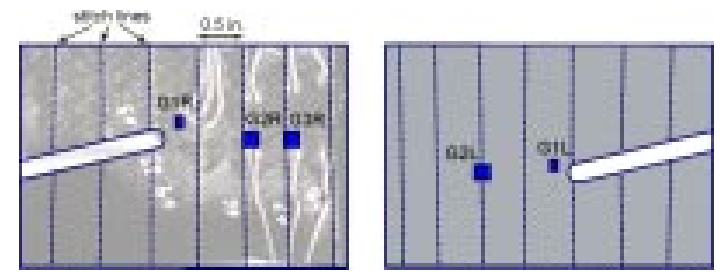

Figure 9. Detail of the notch tips showing strain gage locations in panel P-15.

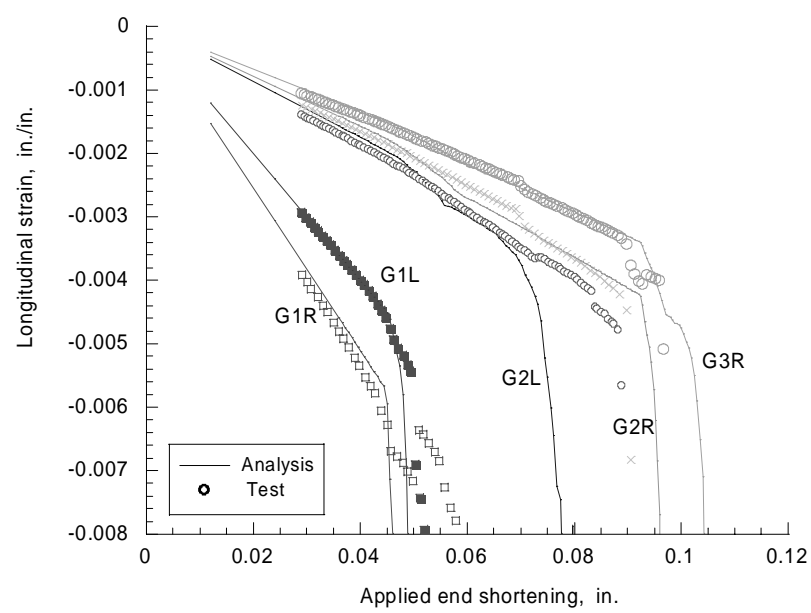

Figure 10. Predicted and measured strains near the notch tips.

The out-of-plane deformation near the center of panel P-15 was measured with displacement transducers. As can be observed in Fig. 11, the panel initially bows out until both the applied end shortening and the out-of-plane deflection reach approximately 0.095 inches. Then, as the damage propagates, the panel cannot maintain its curvature and it begins to flatten out. It can also be observed that the analysis results correlate well with the experimental results. 


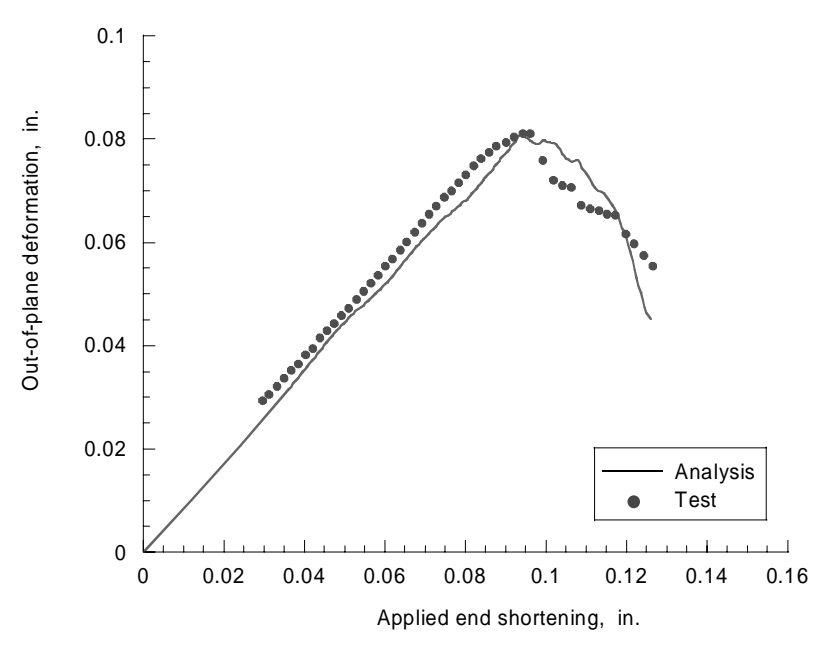

Figure 11. Comparison of analytical and experimental out-of-plane displacement results.

The light-colored area in Fig. 12 represents the 0degree fiber-shearing damage zone at the material points closest to the stiffened side of the surface of the skin for an applied end shortening displacement of 0.08 inches. The strain contours shown in Fig. 13 indicate that the region with high strains is significantly broader than the damage zone. It is noteworthy that the presence of shear in the slanted-notch panels causes the damage zone and the high-strain regions to propagate in a direction that is not exactly normal to the load direction. The damage progresses at an angle of approximately 5 degrees to the panel width direction, and the experimental observation confirms this numerical prediction.

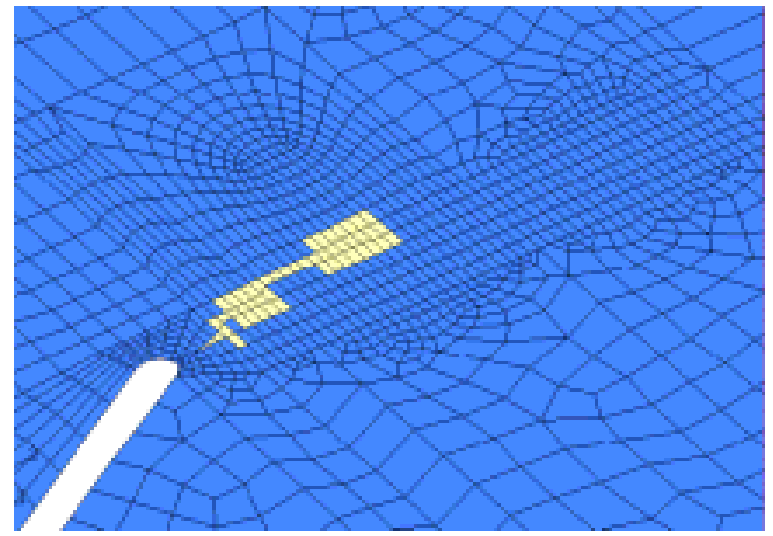

Figure 12. Damage zone for panel P-15 at an applied end shortening displacement of 0.08 inches.

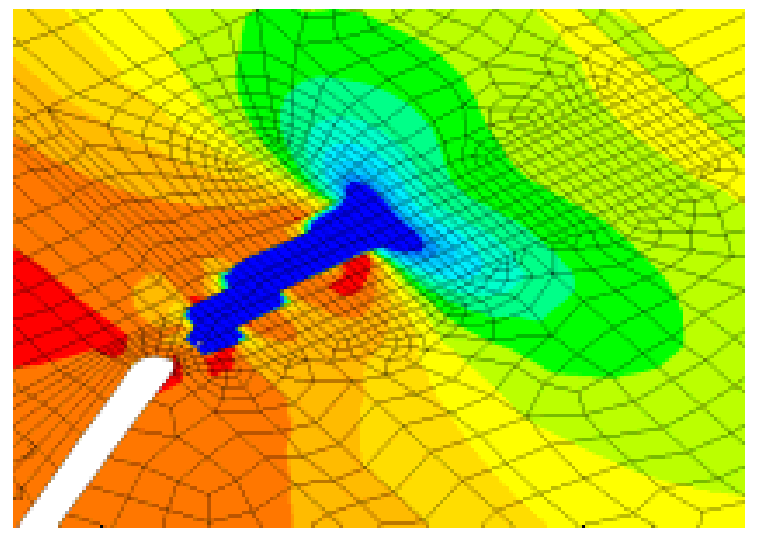

Figure 13. Strain contours for panel P-15 at an applied end shortening displacement of 0.08 inches.

The reaction force for panel P-15 is shown in Fig. 14 as a function of the corresponding applied endshortening displacement. Excellent agreement between analysis and experimental results is achieved for all loads. However, the analysis did not converge at $99 \%$ of the collapse load due to the appearance of negative eigenvalues related to deformations in the damage zone.

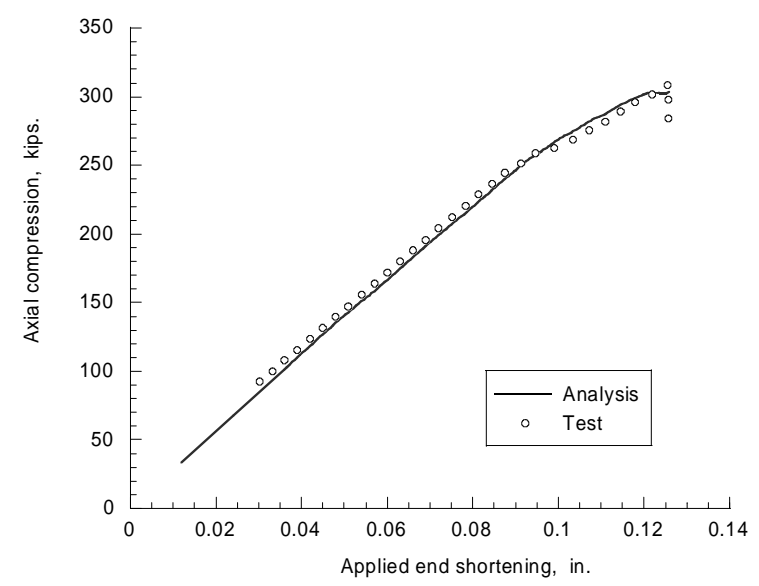

Figure 14. Reaction force as a function of the applied end shortening displacement.

\section{Panel P-30}

Panel P-30 is nominally identical to panel P-15 except for the angle of the notch, which is 30 degrees from the panel width direction, instead of 15 degrees. The strains at gages G1L and G1R are shown in Fig. 15 for both test and analysis. 


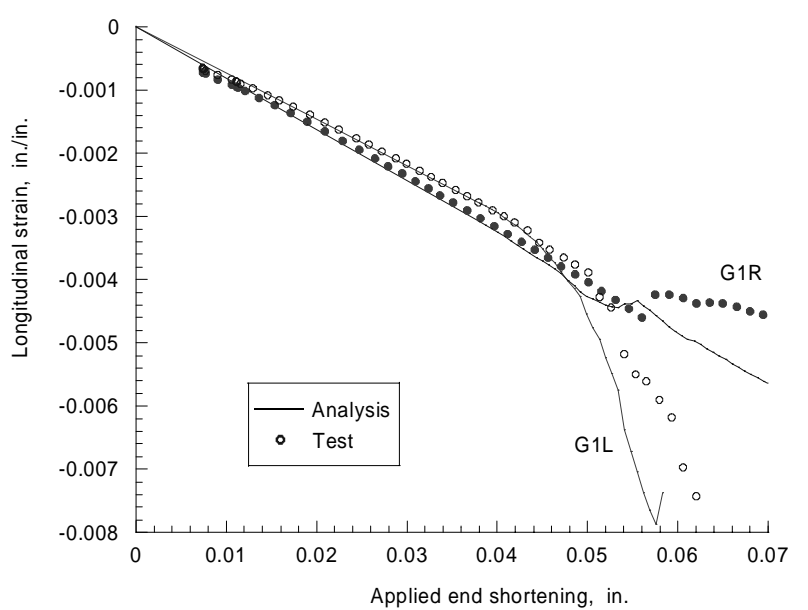

Figure 15. Axial strains at gages G1L and G1R near the notch tips of panel P-30.

The out-of-plane deformation contours for panel P30 are shown in Fig. 16. As with panels P-0 and P-15, panel P-30 initially bows out until both the applied end shortening and the out-of-plane deflection at the center of the panel reach approximately 0.10 inches. The outof-plane displacement is plotted in Fig. 17 as a function of the applied end shortening displacement. As the damage propagates and approaches the stiffener flange, the panel rapidly changes from a curved to a flat configuration. Again, the results predicted by the analysis correlate well with the experimental results up to an end shortening displacement of approximately 0.12 inches. At 0.12 inches of end shortening displacement, the damage zone reaches the stiffener flange, which disbonds as the damage zone grows under the flange. Since delamination and disbond failure mechanisms have not been included in this analysis, discrepancies between the predicted and the experimental results can be expected after this point.

The damage zone corresponding to the fiber-shear failure mode is shown in Fig. 18a. The stations A through E correspond to stitch locations, and station $\mathrm{E}$ is also at the edge of the stiffener flange. Station $F$ is at the center of the stiffener. The growth of the damage zone as a function of the applied end shortening displacement is shown in Fig. 18b. It can be observed that the damage initiates at about 0.046 inches of applied end shortening displacement, and grows 0.2 inches up to the first stitch line (station A). The stitch line at station A arrests the damage until the end shortening displacement approaches 0.08 inches, at which time the damage quickly grows to the next stitch line. The stitches at stations B, C, and D briefly arrest the growth of the damage zone, but their effectiveness is markedly less than that of station A.

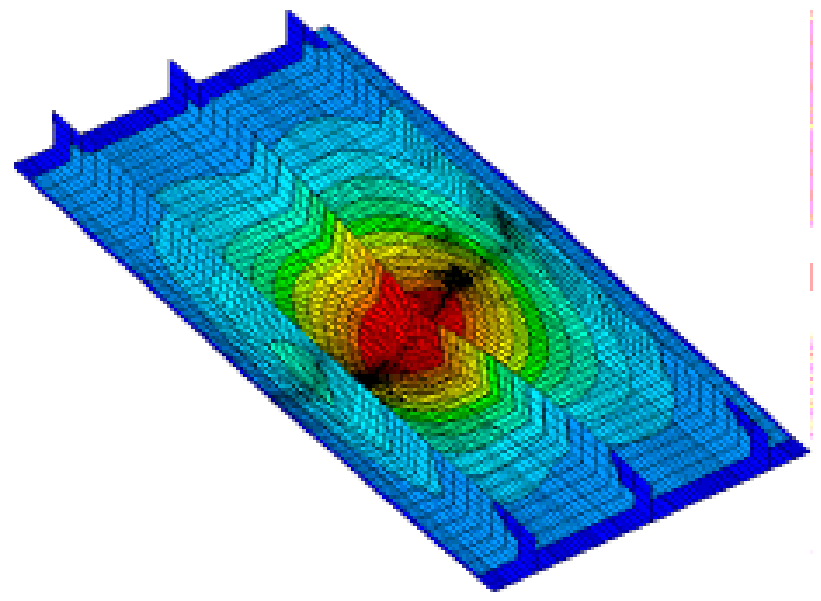

Figure 16. Typical out-of-plane deformation contours for panel P-30.

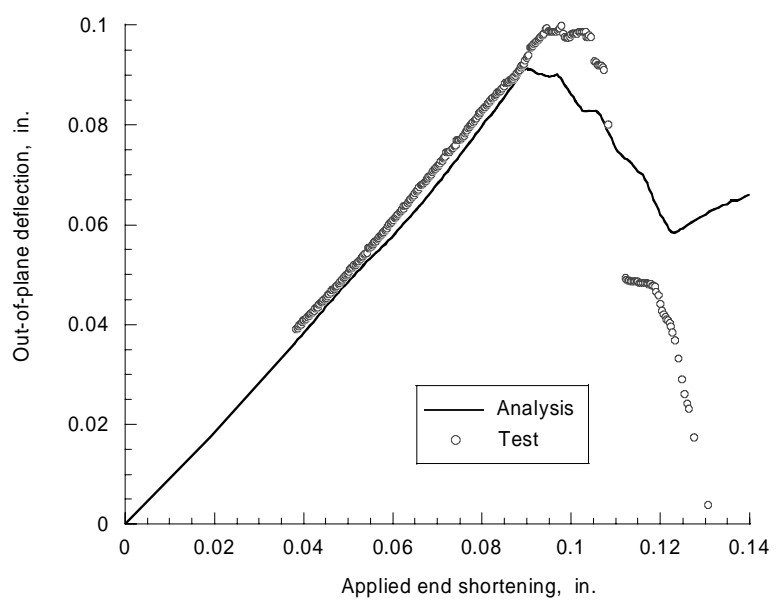

Figure 17. Comparison of analytical and experimental out-of-plane deflection results at the center of panel P-30.

The open circles in Fig. 18b correspond to the approximate position of the damage front during the experiment. The position of the damage front was obtained from a high-resolution video recording of the test. In the video recording, a change in the reflected light was used to determine the approximate position of the damage front. As was observed previously, the agreement between the predicted position of the damage zone and the experimental results is excellent until the damage zone reaches the stiffener flange. After this point, damage growth is a combination of skin failure and disbonding of the stiffener flange from the skin. The discrepancy between the experimental and analytical results between stations $\mathrm{E}$ and $\mathrm{F}$ is attributed to disbonding, which is not included in the present analysis. 


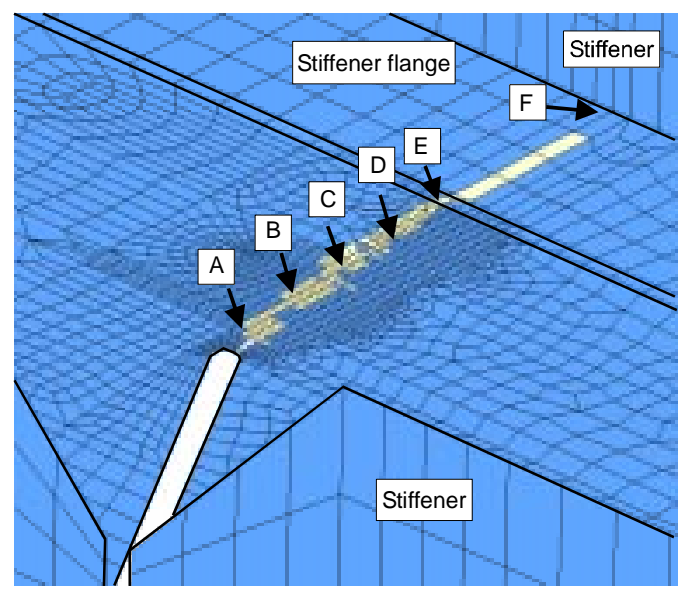

a. Predicted damage zone and stitch line locations at stations $\mathrm{A}, \mathrm{B}, \mathrm{C}, \mathrm{D}$, and $\mathrm{E}$.

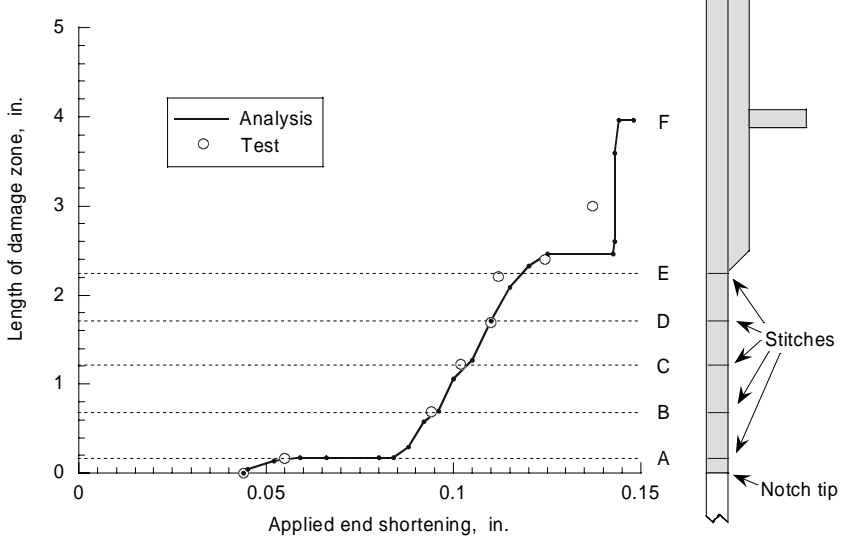

b. Comparison of experimental and predicted position of the front of the damage zone.

Figure 18. Position of the front of the fiber-shear damage zone as a function of the applied end shortening.

The predicted and measured reaction forces as a function of the applied end shortening are shown in Fig. 19. Stations A through F, which were previously described in Fig. 18, are shown for reference. These results indicate that the analysis is unable to predict the proper damage progression mechanism after the damage zone reaches the stiffener flange (station E). One method that would allow the simulation to continue past the initiation of disbonding would be based on the use of a decohesion element that connects the skin to the stiffener flange. Such an element would tie the shell elements of the skin to those of the corresponding stiffener flange using nonlinear material properties.

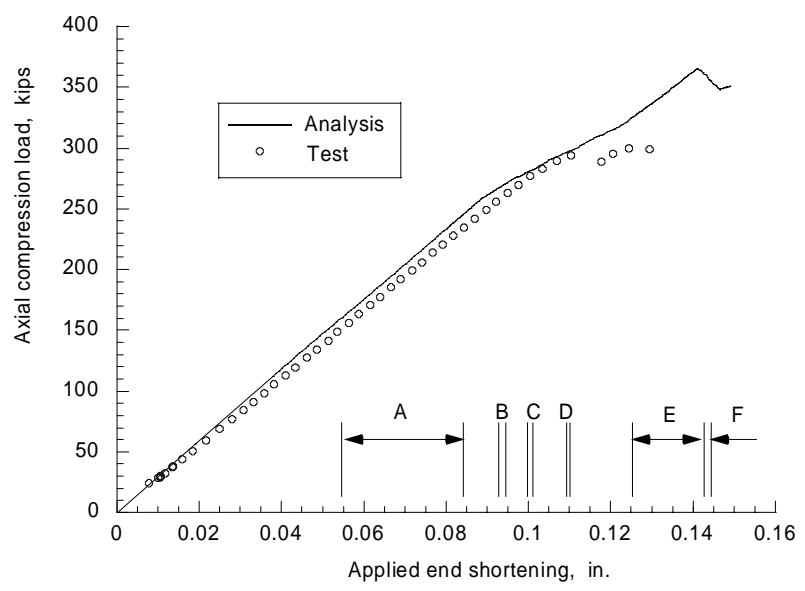

Figure 19. Predicted and measured reaction forces as a function of the applied end shortening.

\section{Concluding Remarks}

A progressive failure analysis methodology was presented for the step-by-step simulation of damage growth in large stiffened stitched composite wing-box cover panels. Three failure indices corresponding to all of the major composite laminate failure modes except delamination were used to evaluate the failure mode and location. Superposed layers of shell elements with multiple integration points through the thickness were used to separate the failure modes for each ply orientation and to obtain the correct effect of bending loads on damage progression. All of the plies of any particular ply orientation were smeared together and combined into one shell element. This method of superposition eliminates the computational expense associated with the otherwise necessary process of evaluating the failure criteria at every integration point in every ply. The present methodology was demonstrated to be accurate and sufficiently efficient to model and analyze relatively large structural components with small load steps in the analysis and a fine mesh in the model.

Three panels with different notch orientations were tested and analyzed in this investigation. The strains, reaction loads, deformations, and damage growth histories were predicted and found to be in excellent correlation with the experimental results. The stitches were found to retard the growth of damage by delaying the onset of fiber buckling in the 0-degree plies. Delamination was not included as a failure mode in the 
present analysis. The stitches and the thickness of the laminates may have contributed to the accuracy of the solution by minimizing delamination as a significant damage mode in the skin. However, the present analysis approach does not model the disbond between the stiffener flange and the skin and, hence, the predicted results were found to become less accurate after the damage zone reached the stiffener flange.

\section{References}

1. Jegley, Dawn C. and Bush, Harold G., "Structural Test Documentation and Results for the McDonnell Douglas All-Composite Wing Stub Box," NASA TM 110204, April 1997.

2. Hinrichs, Steven, General Methods for Determining Stitched Composite Material Stiffnesses and Allowable Strengths, Vol. 1, McDonnell Douglas Report No. MDC94K9113, March 1995.

3. Hashin, Z., "Failure Criteria for Unidirectional Fiber Composites," Journal of Applied Mechanics, Vol. 47, 1980, pp. 329-334.

4. Chang, F.-K., and L.B Lessard, "Damage Tolerance of Laminated Composites Containing an Open Hole and Subjected to Compressive Loadings: Part I-Analysis," Journal of Composite Materials, Vol. 25, 1991, pp. 2-43.
5. Anon., ABAQUS User's Manual, Vol. III, Version 5.6, Hibbitt, Karlsson \& Sorensen, Pawtucket, RI, 1996, p. 25.2.33-1.

6. Anon., ABAQUS Example Problems Manual, Vol. I, Version 5.5, Hibbitt, Karlsson \& Sorensen, Pawtucket, RI, 1995, p. 3.2.25.25-1.

7. Chang, F.-K., and Chang, K.Y., "A Progressive Damage Model for Laminated Composites Containing Stress Concentrations," Journal of Composite Materials, Vol. 21, 1987.

8. Moas, E., "Progressive Failure Analysis of Laminated Composite Structures," Ph.D. Dissertation, Virginia Polytechnic Institute and State University, Blacksburg, VA, 1996.

9. Averill, R.C., "A Micromechanics-Based Progressive Failure Model for Laminated Composite Structures," Proceedings of the AIAA/ASME/ASCE/AHS/ASC $33^{\text {rd }}$ Structures, Structural Dynamics and Materials Conference, Dallas, Texas, April 13-15, 1992.

10. Sleight, D.W., Knight, N.F., Jr., and Wang, J.T., "Evaluation of a Progressive Failure Analysis Methodology for Laminated Composite Structures," Proceedings of the $38^{\text {th }}$ AIAA/ASME/ASCE/ASC Structures, Structural Dynamics, and Materials Conference, Kissimmee, FL, AIAA Paper No. 97-1187, April 7-10, 1997. 